\title{
ABSTRACTION AND CONTROL OF MULTI-DOMAIN DISAGGREGATED OPENROADM OPTICAL NETWORKS
}

\author{
Ramon Casellas ${ }^{1}$, Ricardo Martinez ${ }^{1}$, Ricard Vilalta ${ }^{1}$, Raul Muñoz ${ }^{1}$ \\ ${ }^{1}$ CTTC/CERCA, Optical Networks and Systems Department, Castelldefels, Barcelona, Spain \\ ramon.casellas@cttc.es
}

Keywords: Multi-Domain Optical Network Control, Software Defined Networking, OpenROADM device model.

\begin{abstract} abstraction and control of such networks in line with the ACTN framework.

\section{Introduction}

Several models are possible within the disaggregation trend in optical transport networks, allowing more flexible, reconfigurable and elastic architectures [1], such as a partial disaggregation based on white boxes, where different optical network elements (such as ROADMS [2][3], transponders, line amplifiers, etc.) can be provided by different vendors. Additionally, and in order to scale, operators often segment their networks into domains, and the control of multi-domain transport networks commonly relies on hierarchical control models with a hierarchy of controllers [4]. Finally, the need virtualize the transport network has emerged as a key requirement to support functions such as network slicing and empower end users or to control the allocated partitions [5][6][7]. In this regard, questions related to the efficient and optimal abstraction of the underlying networks or how to support virtualization and slicing remain open.
\end{abstract}

In this paper, we experimentally validate a control plane architecture for multi-domain disaggregated transport networks that relies on the deployment of network elements compliant with the OpenROADM MSA device model. We demonstrate the

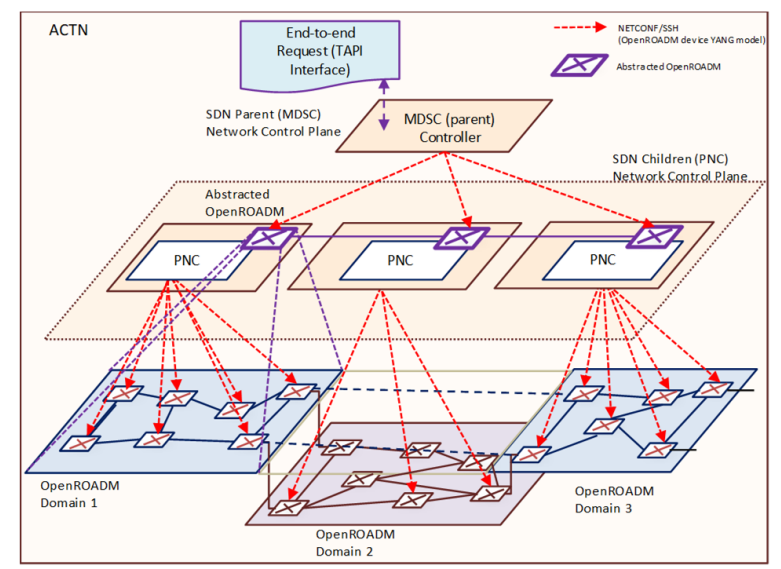

Fig. 1 Architecture based on ACTN and OpenROADM

The IETF Abstraction and Control of Traffic Engineering Networks (ACTN) [8] defines the requirements, use cases, and an SDN-based architecture, relying on the concepts of network and service abstraction, detaching the network and service control from the underlying data plane. The architecture encompasses Physical Network Controllers (PNCs), responsible for specific technology and administrative domains, orchestrated by Multi-Domain Service Coordinator (MDSC) which, in turn, enables underlay transport resources to be abstracted and virtual network instances to be allocated to customers, under the control of a Customer Network Controller (CNC). In this paper, we consider the allocation of network connectivity services (network media channels) across a multi-domain disaggregated optical network, using the ACTN framework while relying on the OpenROADM device model for scalable abstraction (see Fig.1).

\section{Control Plane Architecture}

The generic ACTN does not enforce a given protocol and can be applied or mapped to existing entities, interfaces and protocols, adopting or supporting existing deployments. A particular example is the Path Computation Element (PCE) and PCEP protocol [9]. In this paper, we implement and demonstrate the ACTN approach for a multi-domain OpenROADM network in which the same unified open data model is used to abstract a domain, enabling an arbitrary hierarchy. Internal connections within abstracted ROADMs $(a R O A D M s)$ are mapped to intra-domain (Network) Media Channels or (N)MC.

\subsection{OpenROADM device model}

The OpenROADM [10] device model (v2.2) is used as the basis to perform (domain) abstraction and partitioning. In addition to physical devices, the same YANG data model is used to represent abstracted ROADMs that represent a domain and virtualized ROADMs that are allocated within a network slice (see Fig.2, left). A ROADM is composed of a given number $(\mathrm{N})$ of directions or degrees (DEG) and a given number of add/drop stages (Shared Risk Groups or SRGs in OpenROADM terms). A degree has in and out amplifiers and a WSS to mux/demux the signals towards other degrees or towards add/drop stages. The YANG module defines a first section related to the device information followed by a section that includes a list of circuit-packs, component ports and naming, as well as the correspondence in terms of actual racks and shelves. The model next details the set of ROADM interfaces, internal links, physical links (links between different components, such as a link between Degree 1 and 2, or the drop link between Degree 3 and SRG1) and external 

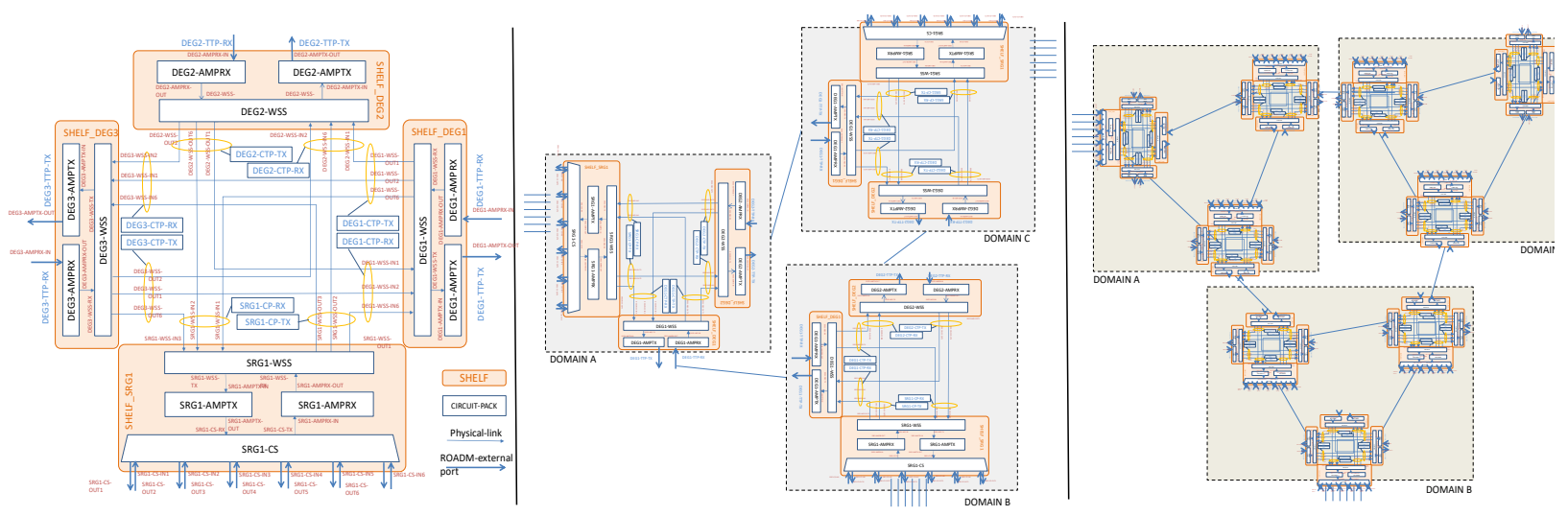

Fig. 2 OpenROADM model (left) and common abstractions: virtual node (center) and virtual link full mesh (right)

links (between ROADMs). Next, the model also includes two lists for the main component blocks: a list of Degrees, and a list of SRGs (Add / Drop groups), with a list of add drop port pairs. Finally, the roadm-connection list includes the connections that are active (established) in the device. Note that the creation of a cross-connection involves creating (logical) interface data nodes within the device, followed by the creation of the actual connection data data node which, once created, is configured in the underlying hardware.

Topology Management and Abstraction Models: each domain controller (PSC) is responsible for abstracting its domain as a (set of) device(s), in such a way that the parent controller (MSDC) manages an abstracted network. Each PSC obtains and manages the domain topology by querying the device (including OpenROADM external links) and manages services instantiated in its domain mapping configuration operations requested on the set of abstracted devices to configuration operations over the underlying physical devices. The MDSC manages domain-connectivity and endpoint reachability. Common abstraction models rely on either a virtual node, a mesh of virtual links or an optimized layout of virtual links according to a per-domain abstraction optimization objective function. In the Virtual Node case (Fig.2. center), an abstracted
OpenROADM device represents the underlying domain connectivity. By design, such OpenROADM device has as many SRGs as the SRGs in the domain, and as many degrees as inter-domain links. In the Virtual Link Mesh, a given set of links model the internal domain connectivity. With a full mesh $\left(\mathrm{N}^{2}\right)$ between edge nodes, the abstraction introduces new edge nodes that have the same number of SRGs and as many DEG elements as (virtual) links towards edge nodes (Fig. 2. right).

\section{Control Plane Workflow}

The provisioning of an end-to-end NMC involves an interaction between the MDSCs and the PNCs using abstracted multi-domain topology, including an optimal domain selection and subsequent segment expansion. After the path computation is complete, the MDSC coordinates the provisioning across the domains. To instantiate an end-to-end service, each PSC entity maps configuration requests coming from the MDSC, defined over the abstracted ROADM (aROADM) into an intra-domain connection supported over several physical ROADM cross-connections within the underlying domain, creating the appropriate interfaces accordingly. For example, an ADD connection in the domain aROADM involves creating $\mathrm{MC}$ and $\mathrm{NMC}$ interfaces in the

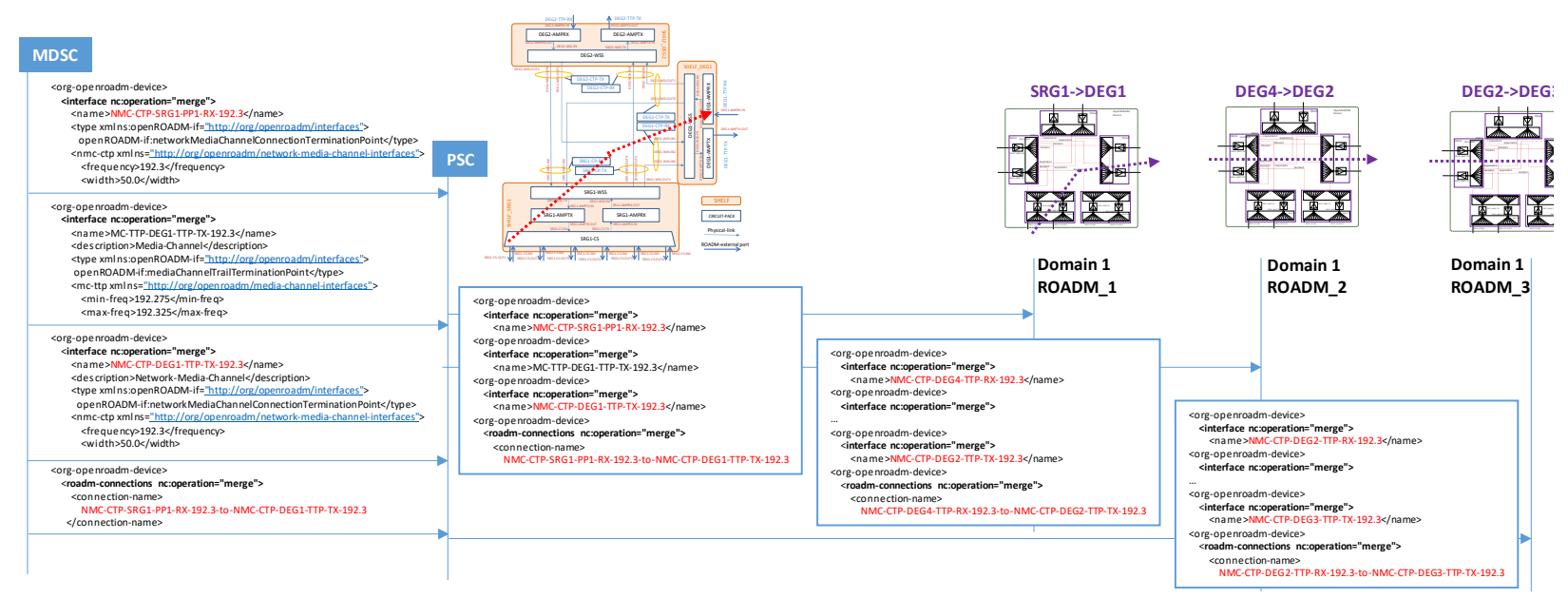

Fig. 3 NETCONF messages for the provisioning of a connection across a domain. MSDC $\rightarrow$ PNC and PNC $\rightarrow$ devices 


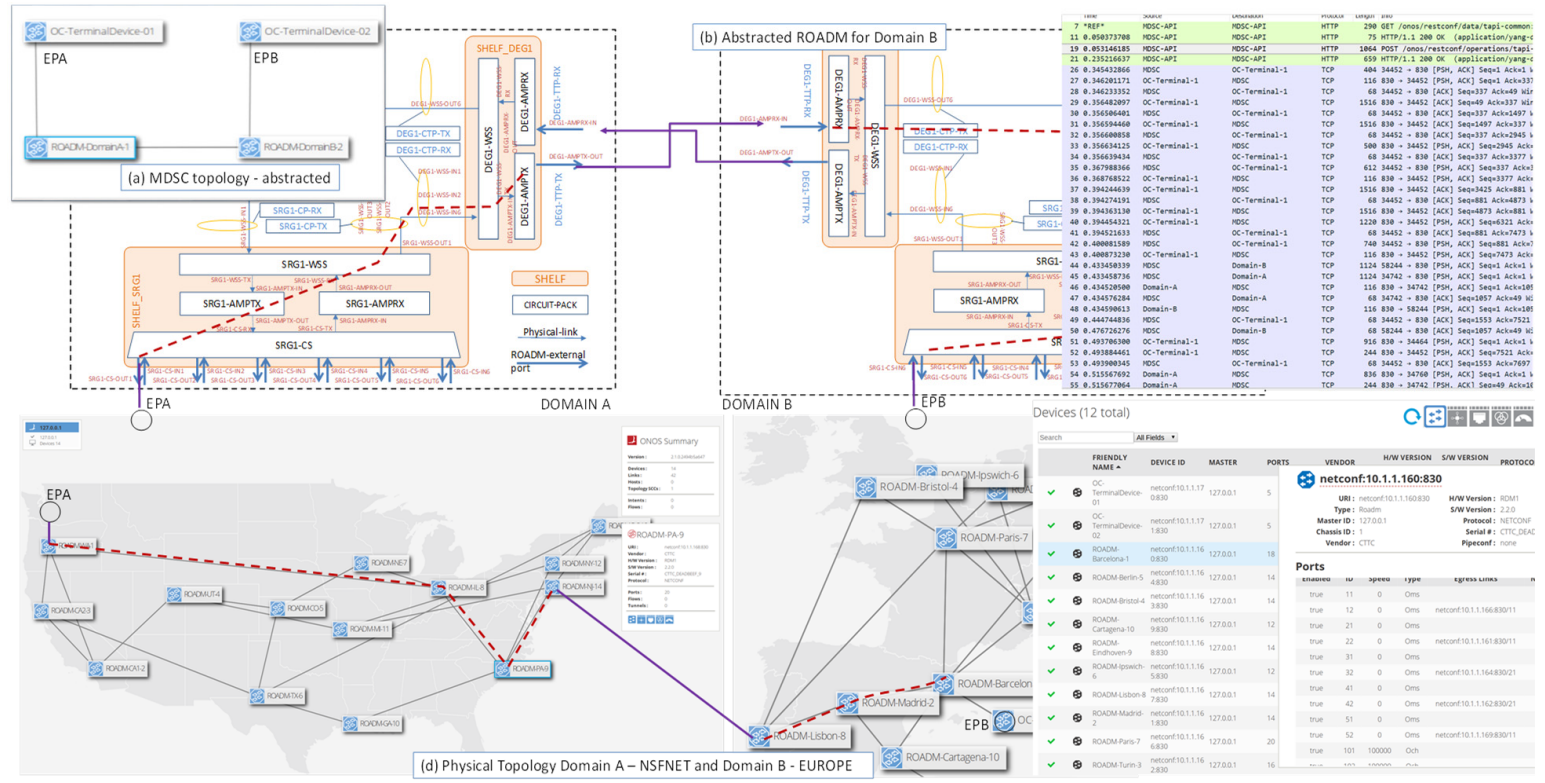

Fig. 4 Experimental evaluation scenario, including abstracted (MDSC) and real (PSC) domain topologies.

device and connecting NMC interfaces. Such operations are mapped into one ADD connection and several EXPRESS ones, each involving the creation of several interfaces and the respective connection objects (see Fig. 3). Finally, Virtual Network Virtualization and Network Slicing support, we rely on using device and network hypervisors, as presented in [7].

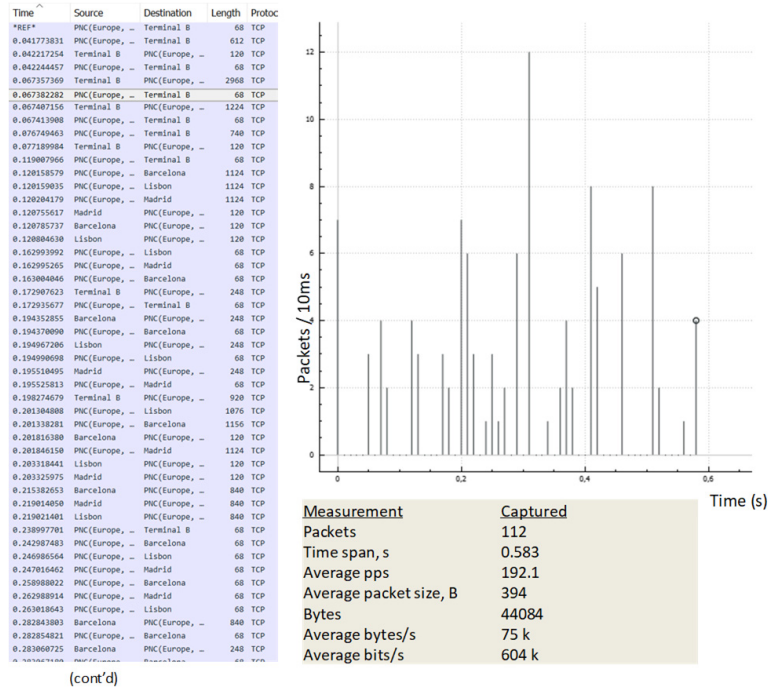

Fig. 5 Capture and throughput in Domain B (Europe).

\section{Experimental Evaluation}

The system has been implemented and demonstrated in a control plane testbed. The MSDC is an ONOS instance extended with OpenROADM drivers [11][12] and a TAPI v2.1 NBI [13] covering the photonic media layer. The PSC controllers combine an application based on ConfD [14] providing a NETCONF frontend and interacting with the ONOS NBI for intra-domain topology abstraction and connectivity configuration. Physical OpenROADMs and
Terminal Devices Transceivers agents are also implemented in ConfD (hardware is emulated). An end-to-end request for a network media channel is requested across 2 domains (Fig. 4), with the 14 node NSFNET and a 10 node Metro-Haul [15] topology. The end-to-end provisioning starting the TAPI connectivity request is received (Fig.4.) is of the $\mathrm{O}(800 \mathrm{~ms})$, including the parallel provisioning within the 2 domains. The cross-connection in the Europe (Dom B) abstracted node is mapped to 3 cross-connections in nodes Lisbon Madrid Barcelona, as shown in Fig. 5. Within the domain, the provisioning is $\mathrm{O}(500 \mathrm{~ms})$. Note that there is an overhead, given the XML encoding, the SSH transport for Netconf and the need to send multiple messages (creation of MC, NMC interfaces, and connections, OMS and OTS interfaces at each degree port are pre-created). As per Fig. 5, there are O(100) IP packets exchanged between PNC and agents, but the overall throughput is relatively low, being latency the critical aspect. Setup delays are computed without taking into account hardware configuration delays with real ROADMs [11], which can be on the order or seconds/minutes. Setup delay due to control plane only has little impact on the performance.

\section{Conclusion}

We have experimentally validated a control plane for multidomain optical networks based on the ACTN framework and the OpenROADM device model, used in the abstraction of each domain and the actual devices. An end-to-end network media channel has been demonstrated hierarchically, using TAPI NBI, requested between transponders client ports. The delay and control plane throughout is within targeted values, validating the proposed approach.

\section{Acknowledgements}

This work has been partially supported by the EU-H2020 Metro-Haul Project with G.A 761727 and the Spanish AURORAS (RTI2018-099178) project. 


\section{References}

[1] Riccardi, E., Gunning, P, González de Dios, et al., 'An Operator view on the Introduction of White Boxes into Optical Networks', Journal of Lightwave Technology, 2018, 36, (15), pp. 3062-3072

[2] Calabretta, N., Prifti, K., Tessema, N., et al., 'Photonic integrated WDM cross-connects for optical metro and data center networks', in Proceedings of Metro and Data Center Optical Networks and Short-Reach Links II, February 2019.

[3] Kundrát, J., Havlis, O., Jedlinsky, J., et al. "Opening up ROADMs: Let's Build a Disaggregated Open Optical Line System". Journal of Lightwave Technology. PP. 11. 10.1109/JLT.2019.2906620, 2019

[4] Casellas, R., Martinez, R., Vilalta, R., and Munoz, R., "Control, management, and orchestration of optical networks: Evolution, trends, and challenges," Journal of Lightwave Technology, vol. 36, no. 7, pp. 1390-1402, 2018

[5] Channegowda, M., Nejabati, R., Simeonidou, D. "Software-defined optical networks technology and infrastructure: Enabling software-defined optical network operations", Journal of Optical Communications and Networking, 5, (10), A274-A282, 2013

[6] Li, X., Casellas, R., Landi, G. et al, "5G-Crosshaul Network Slicing: Enabling Multi-Tenancy in Mobile Transport Networks", IEEE Communications Magazine, special issue on Network Slicing in 5G systems, August 2017, Vol. 55, No. 8, August 2017.

[7] Casellas, R., Giorgetti, A., Morro, R., et al "Enabling Network Slicing Across a Disaggregated Optical Transport Network", in Proceedings of the Optical Networking and Communication Conference \& Exhibition (OFC), 3-7 March 2019, San Diego, CA (USA). 2019.

[8] Ceccarelli, D., Lee, Y., editors, "Framework for Abstraction and Control of TE Networks (ACTN)", IETF RFC8453, august 2018.

[9] Casellas, R., Vilalta, R., Martínez, et al "Experimental Validation of the ACTN architecture for flexi-grid optical networks using Active Stateful Hierarchical PCEs", in Proc. 19 International Conference on Transparent Optical Networks (ICTON2017), 2-6 July, 2017, Girona, Spain, July 2017.

[10] The Open ROADM Multi-Source Agreement (MSA) http://www.openroadm.org Accessed: 2019-04-12

[11] Morro, R., Lucrezia, F., Gomez, P., et al., "Automated End to End Carrier Ethernet Provisioning over a Disaggregated WDM Metro Network with a Hierarchical SDN Control and Monitoring Platform", in Proceedings of 44th European Conference on Optical Communication (ECOC 2018), 23-27 September 2018, Roma (Italy).

[12] Giorgetti, A., Casellas, R., Morro, R., et al., "ONOScontrolled Disaggregated Optical Networks", in Proceedings of the Optical Networking and Communication Conference \& Exhibition (OFC), 3-7 March 2019, San Diego, CA (USA). 2019
[13] Open Networking Foundation (ONF), Transport API project https://wiki.opennetworking.org/display/OTCC/TAPI Accessed: 2019-04-12

[14] ConfD, management agent software framework for network elements https://www.tail-f.com/confd-basic/ Accessed: 2019-4-12.

[15] The Metro-haul project http://metro-haul.eu 